\title{
A study on prescribing pattern of antibiotics in medicine ward of tertiary care teaching hospital
}

\author{
Akhilesh Patel*, Mamta Naagar, Rajveer Singh
}

Department of Pharmacy Practice, NIMS University, Jaipur, Rajasthan, India

\author{
Received: 11 February 2020 \\ Revised: 05 April 2020 \\ Accepted: 09 April 2020 \\ *Correspondence: \\ Dr. Akhilesh Patel, \\ Email: akhileshpate10112@gmail.com
}

Copyright: $@$ the author(s), publisher and licensee Medip Academy. This is an open-access article distributed under the terms of the Creative Commons Attribution Non-Commercial License, which permits unrestricted non-commercial use, distribution, and reproduction in any medium, provided the original work is properly cited.

\begin{abstract}
Background: Antibiotics are most frequently prescribed and problem associated with antibiotics over and under use leads to treatment failure and on the other side resistance to drugs increases. Assessment of antibiotics prescribed is important for checking quality and standard of prescriptions. The objective of the study was done for assessing the prescribing pattern of antibiotics using WHO indicator in medicine ward of tertiary care teaching hospital.

Methods: Prospective observational study was carried out for a period of six months in medicine ward after ethical committee approval. Data was collected in data collection forms and evaluated using WHO indicator.

Results: A total of 190 prescriptions was collected in which 1145 drugs were prescribed. Total number of antibiotics in prescription was found to be 164. Percentage of antibiotics was found to be $14.32 \%$ which was according to WHO prescribing indicator. Various class of antibiotics was prescribed in which cephalosporin with total of $90(54.87 \%)$ followed by penicillin 18 (10.97), macrolide 18 (10.97\%) fluoroquinolones 11 (6.70\%), nitroimidazole 8 (4.87\%), amino glycoside $8(4.87 \%)$ and other classes $11(6.70 \%)$.

Conclusions: The study shows that pattern of antibiotics was according to the WHO prescribing indicator but still practice was lacking in terms of selection of antibiotics as there was no culture sensitivity test was performed.
\end{abstract}

Keywords: WHO indicator, Antibiotics, Antibiotics resistance, Rational use

\section{INTRODUCTION}

In medical practice drugs prescribing is crucial and it's directly affects patient belief to health care system. Prescribing drug is totally depending on the practitioner training and attitude towards the cure of a disease. Different factors affect for irrational prescribing of drugs. Thousands of drug manufacturer companies using different brand names for selling products in market. Irrational prescribing causes improper and over use of drugs. Drug interaction, side effects and adverse drug reaction are common problem associated with irrational prescribing. Over use of antibiotics leads to treatment failure, and antibiotic resistance..$^{1-4}$
Precise use of antibiotics important in terms of patient health and benefits. Drug use can be rationalized by proper implementation of prescribing guidelines, essential medicine list and formulary. It requires selection of medication according to need of the patients, proper dose selection, proper route of administration, cost of therapy and adequate time period of antibiotics use. Drug utilization review (DUR) helps in better practice and better patient care..$^{5-7}$

Generally, dispenser, prescriber, patient and community are responsible for irrational use of medicines. In such condition it's become important to assess various problems and prepare interventional strategies for them. INRUD and WHO forms three different component of 
core indicators are Prescribing indicators, patient care indicators and facility indicators. ${ }^{8,9}$ Prescribing indicators are: average number of drugs prescribed, percentage of drug by generic name, percentage of antibiotics prescribed, percentage of drug form essential medicine list, and percentage of encounters with an injection prescribed. ${ }^{10}$

\section{Aim and objectives}

The present study was conducted with an aim to prepare data regarding antibiotic prescribing pattern and rationality associated with the antibiotic use by using WHO prescribing indicator.

\section{METHODS}

After approval from institutional ethics committee, a prospective observational study was conducted in medicine ward of NIMS Institute of Medical Sciences and Research, Jaipur, Rajasthan, India. Study period was February to July 2019. Patients were enrolled on the basis of inclusion and exclusion criteria and analysed. Data was collected in data collection forms from patient case records. Form contain patient demographic detail, diagnosis, medication chart of patients. Data were analyzed using WHO indicator and presented using descriptive statistics namely total numbers, mean and percentage wherever applicable.

\section{RESULTS}

Total of 190 prescription was collected from inpatient medicine department of tertiary care teaching hospital. Out of 190 prescriptions, $95(50 \%)$ were from male medicine ward and $95(50 \%)$ were from female medicine ward (Figure 1).

\section{Percentage of antibiotics prescribed}

Total of 164 antibiotics prescribed i.e. $14.32 \%$ which was according to WHO indicator. 82 were prescribed in male while 82 were prescribed in female medicine ward (Figure 1).

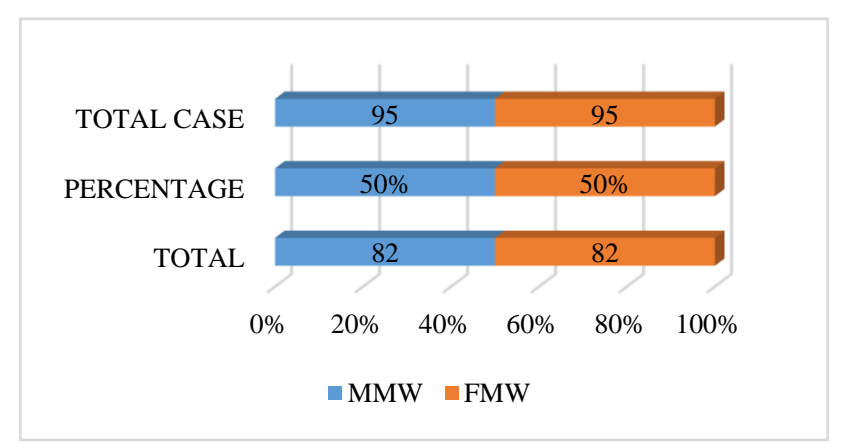

Figure 1: Total number of cases from male and female medicine ward with percentage and total no. antibiotics in male and female medicine ward.
Patient were divided on the basis of age group: $\leq 20$, (21$40)$ years, (41-60) years, (61-80) years and $\geq 81$ and above (Figure 2).

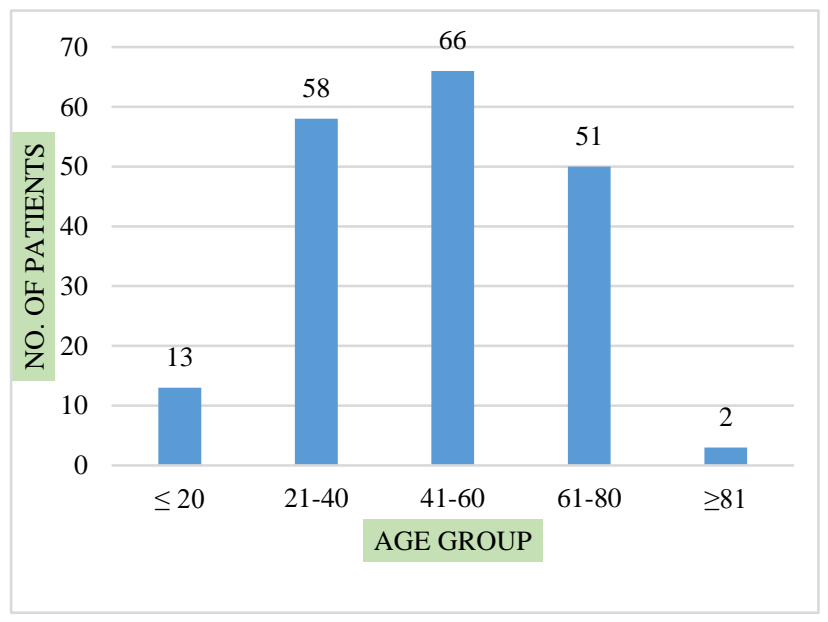

Figure 2: Total number of cases in different age groups.

\section{Different class of antibiotics}

Percentage of different class of antibiotics was found to be cephalosporin $54.87 \%$, penicillin and macrolide $10.97 \%$, fluoroquinolones $6.70 \%$, amino glycoside and nitroimidazole was $4.87 \%$ and other class of antibiotics was $6.70 \%$ (Figure 3 ).

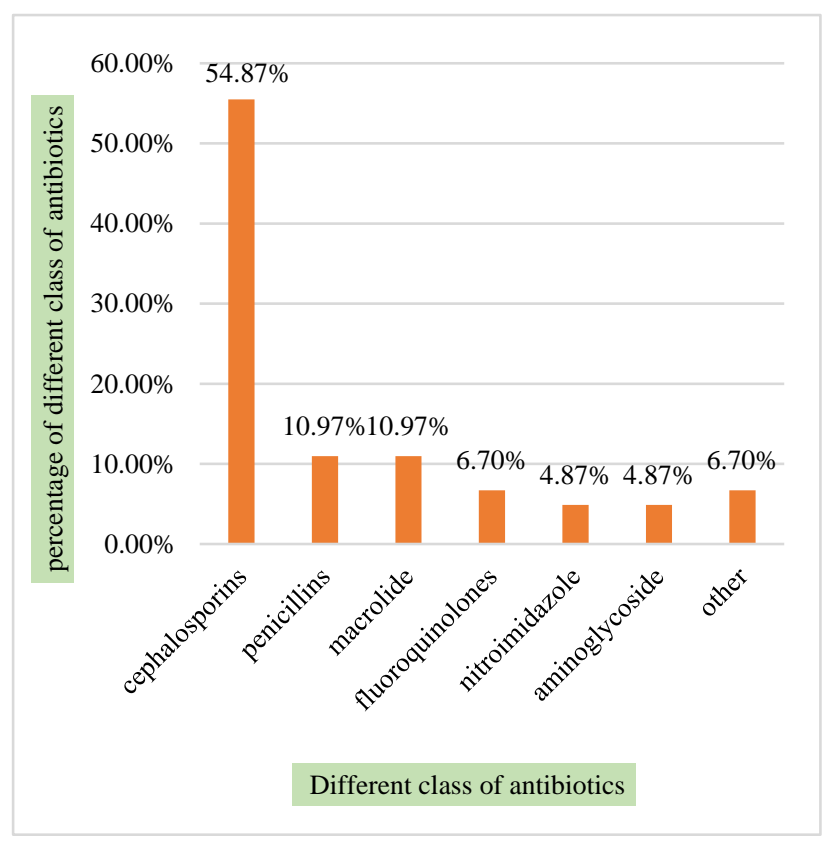

Figure 3: Percentage of different class of antibiotics.

\section{Route of antibiotics administered}

In 164 antibiotics parenterally prescribed drug was found to be $139(84.75 \%)$ followed by oral route $15(9.14 \%)$ and topical route $10(6.09 \%)$ (Figure 4$)$. 


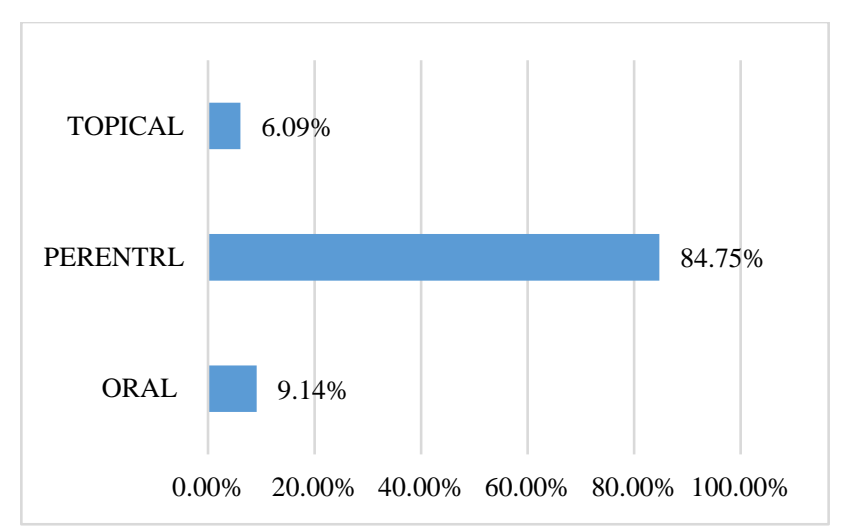

Figure 4: Percentage of different route for antibiotics use.

\section{Pattern of use of antibiotics in study population}

Out of 190 prescription included in study, 62 (32\%) prescription having no antibiotics prescribed, 97 $(51.05 \%)$ having single antibiotics, $27(14.21 \%)$ having two antibiotics, $3(1.5 \%)$ having three antibiotics and 1 $(0.5 \%)$ having four antibiotics (Figure 5).

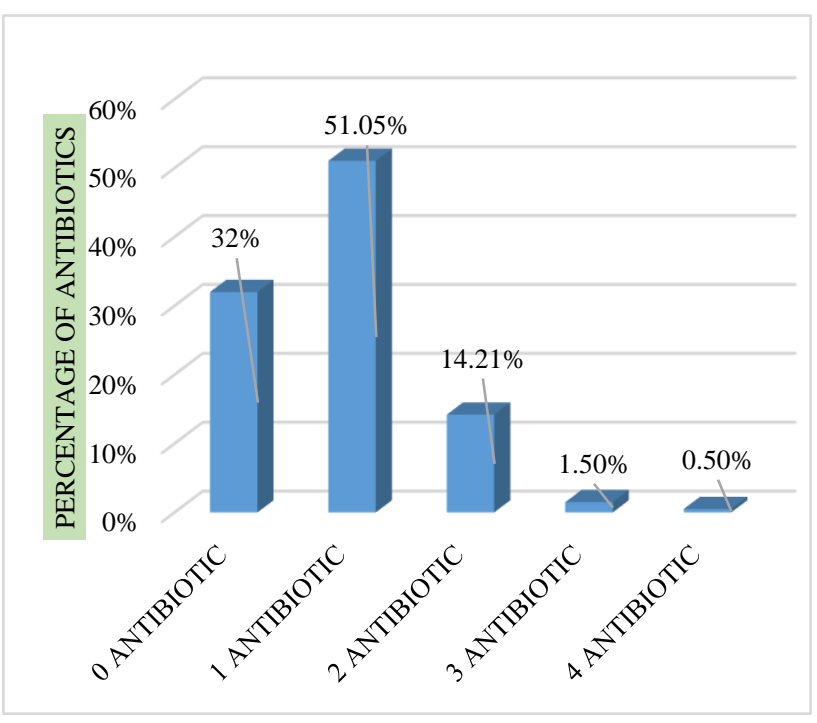

Figure 5: Percentage of number of antibiotics in prescription.

\section{DISCUSSION}

The study shows prescribing practice and the use of antibiotics was according to WHO prescribing indicator and also according to patient needs. This study was valuable in terms of the Indian health care system because it helps in ensuring the proper and optimal use of medication. Besides, our findings may apply to similar drug practices in other health care facilities.

Percentage of antibiotic prescribed was found to be $14.32 \%$ which was lower than the study conducted by Pathak et al, Narendra et al, Ulhas et al, and Durga et al, i.e. $24.64 \%, 78.25 \%, 31.8 \%$ and $46.21 .^{10-13}$ The value is higher than study conducted by Hussain et al, Farzana et al, Bansal et al, and Singh et al, which was around $19.70 \%, 15.95 \%, 18.10 \%$ and $18.5 \% .{ }^{14-17}$ Broad spectrum antibiotics was preferred as major choice of antibiotics. Class of antibiotics includes are cephalosporin, penicillin, aminoglycosides, nitroimidazole, macrolide, fluoroquinolones and other. Selection and choice of drug can be done on the basis of final diagnosis, patient allergic status to drug and culture sensitivity test.

\section{Limitations and strength of study}

As study conducted in single centre outcomes are not applicable to other health facility. Data was collected from medicine department on the basis of selection criteria which limits finding. Lack of local guidelines and evaluation of adherence to such guidelines was also a limitation to study. Different practitioner having different prescribing information and it was not comparable in each IPD units. As strength, outcomes help in understanding the trend as well as proper and rational use of antibiotics.

\section{CONCLUSION}

Timely prescription monitoring and auditing in health care facility helps in assessing the prescribing pattern as well as uses of drugs, and it also provide information regarding the prescribing practice trend and attitude of a practitioner. Multidisciplinary approach by practitioners, staffs, pharmacists and infection control committee promotes the rationality in drug use pattern. Diagnosis and culture sensitivity tests to antibiotics helps in selection of proper antibiotics according to patients need. Information about hospital guidelines, formulary and antibiotics policy should be provided and present at various departments for better outcomes.

\section{ACKNOWLEDGEMENTS}

Author acknowledging Head of Department of Medicine and Institutional Ethics Committee, NIMS Hospital, Jaipur, Rajasthan, for providing support and facilities.

Funding: No funding sources

Conflict of interest: None declared

Ethical approval: The study was approved by the Institutional Ethics Committee

\section{REFERENCES}

1. Kumar S, Agrawal D, Swain TR, Sahu PK. A study of prescribing pattern of antibiotics and other drugs in surgical wards of a teaching hospital in southeast India. Int J Pharma Sci Res. 2018;3(1):12-4.

2. Pallavi PS, Sree BT, Krishnakanth PV. Study of prescription patterns of antibiotics in tertiary care hospital. Int J Biomed Res. 2016;7(6):372-4.

3. Nia SS, Hiremath SRR, Prashad S. Assessment of Antimicrobial Use Pattern Using World Health 
Organization Prescribing Indicators at a Tertiary Hospital: A Prospective, Observational study. J Applied Pharm Sci. 2018;8(6):132-8.

4. Adisa R, Fakeye OT, Aindero OV. Evaluation of prescription pattern and patients' opinion on healthcare practices in selected primary healthcare facilities in Ibadan, South-Western Nigeria. Afri Health Sci Dec. 2015;5(4):1318-29.

5. Karimi A, Haerizadeh M, Soleymani F, Haerizadeh M. Evaluation of medicine prescription pattern using World Health Organization prescribing indicators in Iran: A cross-sectional study. J Res Pharm Pract. 2014;3(2):39-45.

6. Meher BR, Mukharjee D, Shankar U. A study on antibiotic utilization pattern in a general medicine ward of a tertiary care teaching hospital. J Chem Pharm Res. 2014;6(7):1847-9.

7. Ramakrishna P, Ramaiah R, Kumar PK. Monitoring of prescription pattern of antimicrobial agents in medicine department of a tertiary care hospital. Int $\mathbf{J}$ Res. Ayurveda Pham. 2016;7(4):78-82.

8. Demeke B, Molla F, Assen A, Melkam W. evaluation of drug utilization pattern using WHO prescribing indicators in Ayder Referral Hospital, Northern, Ethiopia. Int J Pharma Sci Res. 2015;6(2):343-7.

9. Mensa M, Tadesse T, Ayele A. Assessment of Drug Use Pattern by Using WHO Core Drug Use Indicators at Public Hospitals in Ethiopia. J Community Med Health Educ. 2017;7(5):1-10.

10. Pathak A, Gupta VK, Maurya A, Kumar A. Assessment of drug prescribing pattern using WHO indicators in hospitalized patients at a tertiary care teaching hospital in rural area of India. Int $\mathbf{J}$ Basic Clin Pharmacol. 2016;5(3):651-5.

11. Bachewar NP, Choudhari SR, Dudhgaonkar S. Assessment of prescription pattern using WHO drug prescribing indicators in medicine wards of a tertiary care teaching hospital: a retrospective observational study. Int J Basic Clin Pharmacol. 2017;6(8):2070-7.

12. Chandelkar UK, Rataboli PV. A study of drug prescribing pattern using WHO prescribing indicators in the state of GOA, India. Int $\mathbf{J}$ Basic Clin Pharmacol. 2014;3(6):1057-61.

13. Durga P, Abhinav P, Raj VK, Kishore P. Evaluation of Prescribing Patterns Using WHO Indicators at Out Patient Department of A Private Hospital in Warangal. IOSR-JOPBS. 2017;12(3):1-4.

14. Hussain S, Yadav SS, Sawlani KK, Khattri S. Assessment of Drug Prescribing Pattern Using World Health Organization Indicators in a Tertiary Care Teaching Hospital. Indian J Public Health. 2018;62(2):156-8.

15. Sultana F, Rahman A, Paul TR, Sarwar SM. Prescribing Pattern and Prescription Errors: A Study at a Tertiary Care Hospital of Bangladesh. Bangladesh Pharm J. 2015;18(1):20-4.

16. Bansal A, Jain C, Advani U, Sharma N. Current scenario of common diseases and their management using WHO drug use indicators in pediatric outdoor patient in Jaipur. World J Pharm Res. 2017;6(6):92935 .

17. Singh G, Bhatnagar A, Mukherji S, Goel D. Drug prescription behavior in a Teaching Hospital of Western Maharashtra. Med J. 2016;9(6):695-700.

Cite this article as: Patel A, Naagar M, Singh R. A study on prescribing pattern of antibiotics in medicine ward of tertiary care teaching hospital. Int J Basic Clin Pharmacol 2020;9:887-90. 